\title{
Belatacept, a New Paradigm in Organ Transplantation
}

\section{Wael Lateef Jabur}

Nephrologist, Dubai, UAE

It has been long time before a new medicine introduced to the forefront of immunosuppressant therapies. It has come after long extended studies, enrolling hundreds of patients, from different countries, in the phase III of the widely known BENEFIT, and BENEFITEXT trials. It sounds like a new hope for the transplantation community, which is still striving to overcome the hurdles of the older regimens of immunosuppressant's, with its critical side effects, in particular the nonspecificity of the immunosuppression with the consequent issues of the opportunistic infections, the lymphoproliferative and the neoplastic complications. And the other common nonspecific complications like hypertension, diabetes, and the hyperlipidemia with its subsequent mounting cardiovascular mortality and morbidity (the most common cause of mortality in transplant recipients). And most importantly, the ever challenging chronic allograft nephropathy which is partly attributed to the direct Calcineurin inhibitors (CNI) toxicity. In the other hand, there was the debatable issue of delayed graft function, and the slow allograft recovery, which might be both attributed similarly to the induction phase of immune suppressive regimen including the CNIs. Therefore it has been a long time, of extended deliberations, about several new agents and new suggested regimens to overcome the early and the late deleterious side effects. In parallel was the discovery long back of the co-stimulatory pathway of the T- cytotoxic lymphocytes antigen (CTLA-4), and the apparent inhibition and consequent revoking of the T-lymphocyte mediated immune response, as a new horizon that revolutionized dramatically the researches henceforth and the dream came true with the BENEFIT and BENEFIT -EXTENDED trials with more than 660 and 500 patients enrolled in each, categorized into three groups of kidney transplantation recipients with three different immunosuppressant protocols, first protocol included cyclosporine, mycophenolate mofetil and steroid, second protocol included belatacept low intensity with mycophenolate mofetil and steroid and third group with high intensity belatacept and similarly with mycophenolate mofetil and steroid. Induction with basiliximab was adopted in all groups. Patient and allograft survival were the end points after a period of follow up of one and two years and then beyond the second year. The results after the two periods were comparable for both of the limbs, with the serum creatinin and eGFR were significantly better in the Belatacept groups at one year (eGFR is $15-17 \mathrm{ml} / \mathrm{min}$ more) and then at two years, and better cardiovascular profile. However there has been significantly higher incidence and grade of acute rejections with belatacept based regimen than with cyclosporine based ones, nevertheless the apparent risk of chronic allograft nephropathy was similar in both limbs at the end of the study, a fact that might need more time of scrutiny to delineate. The other caveat of the study is the comparison of belatacept with cyclosporine itself, which is currently very rarely used in kidney transplantation protocols across USA and Europe, and constantly being replaced with the more potent CNI (tacrolimus), a fact that might undermine the credibility of the study. Similarly, enough data to compare the new agent to the already existing agents is still lacking, in particular the debatable mammalian target of Rapa inhibitors (mTORi), with its parallel potential of CNI sparing, and lessened renal toxicity. Lastly it didn't answer the question of whether the new biological agent belatacept, is more steroid sparing then CNIs? More time in the transplantation practice is warranted to answer the ongoing contentious questions.
Corresponding author: Dr. Wael Lateef Jabur MD, Nephrologist, Dubai, UAE, Fax: 0097142678889; E-mail: wael69jabur@yahoo.com

Received August 08, 2011; Accepted November 25, 2011; Published December 15, 2011

Citation: Jabur WL (2012) Belatacept, a New Paradigm in Organ Transplantation J Transplant Technol Res 2:108e. doi:10.4172/2161-0991.1000108e

Copyright: ( 2012 Jabur WL. This is an open-access article distributed under the terms of the Creative Commons Attribution License, which permits unrestricted use, distribution, and reproduction in any medium, provided the original author and source are credited. 